Shinji Kunishima $\cdot$ Tadashi Matsushita $\cdot$ Tetsuhito Kojima

Norihiko Amemiya • Yong Mook Choi • Naoki Hosaka

Masakazu Inoue · Youngzoon Jung • Shigeo Mamiya

Kimikazu Matsumoto · Yuji Miyajima • Guangsen Zhang

Changgeng Ruan · Koki Saito $\cdot$ Kyung Soon Song

Hwi-Joong Yoon · Tadashi Kamiya · Hidehiko Saito

\title{
Identification of six novel MYH9 mutations and genotype-phenotype relationships in autosomal dominant macrothrombocytopenia with leukocyte inclusions
}

Received: October 13, 2001 / Accepted: October 15, 2001

\begin{abstract}
The autosomal dominant macrothrombocytopenia with leukocyte inclusions, May-Hegglin anomaly (MHA), Sebastian syndrome (SBS), and Fechtner syndrome (FTNS), are rare platelet disorders characterized by a triad of giant platelets, thrombocytopenia, and characteristic Döhle body-like leukocyte inclusions. The locus for these disorders was previously mapped on chromosome 22q12.3-q13.2 and the disease gene was recently identified as $M Y H 9$, the gene encoding the nonmuscle myosin heavy chain-A. To elucidate the spectrum of MYH9 mutations responsible for the disorders and to investigate genotypephenotype correlation, we examined MYHO mutations in an additional 11 families and 3 sporadic patients with the disorders from Japan, Korea, and China. All 14 patients had
\end{abstract}

S. Kunishima $\cdot$ T. Matsushita $\cdot$ T. Kojima $\cdot$ H. Saito $(\bowtie)$

First Department of Internal Medicine, Nagoya University School of Medicine, 65 Tsurumai-cho, Showa-ku, Nagoya 466-8550, Japan

Tel. +81-52-744-2136; Fax +81-52-744-2157

e-mail: hsaito@med.nagoya-u.ac.jp

S. Kunishima $\cdot$ T. Kamiya

Japanese Red Cross Aichi Blood Center, Seto, Japan

T. Kojima

Department of Medical Technology, Nagoya University School of Health Sciences, Nagoya, Japan

N. Amemiya

Central Clinical Laboratory, Yamanashi Medical University,

Yamanashi, Japan

Y.M. Choi

Department of Pediatrics, Kyung Hee University Hospital, Seoul, Korea

N. Hosaka

Department of Clinical Pathology, Toyooka Hospital, Toyooka,

Japan

M. Inoue

Department of Pediatrics, Yamada Red Cross Hospital, Mie, Japan

Y. Jung

Department of Clinical Pathology, Halla General Hospital, Seoul,

Korea heterozygous $M Y H 9$ mutations, including three known mutations and six novel mutations (three missense and three deletion mutations). Two cases had Alport manifestations including deafness, nephritis, and cataracts and had R1165C and E1841K mutations, respectively. However, taken together with three previous reports, including ours, the data do not show clear phenotype-genotype relationships. Thus, MHA, SBS, and FTNS appear to represent a class of allelic disorders with variable phenotypic diversity.

Key words Alport syndrome $\cdot$ Chromosome $22 \cdot$ Epstein syndrome $\cdot$ Fechtner syndrome - May-Hegglin anomaly · Nonmuscle myosin heavy chain-A · MYH9 - Sebastian syndrome
S. Mamiya

Third Department of Internal Medicine, Akita University School of Medicine, Akita, Japan

K. Matsumoto

Department of Pediatrics, Toyota Memorial Hospital, Toyota, Japan

Y. Miyajima

Department of Pediatrics, Kosei Hospital, Anjo, Japan

G. Zhang

Xiangya Second Hospital, Central South University, Changsha, China

C. Ruan

Suzhou University Medical College, Suzhou, China

K. Saito

Department of Internal Medicine, Hiraga General Hospital, Yokote, Japan

K.S. Song

Department of Clinical Pathology, Yonsei University Medical Center, Seoul, Korea

H.-J. Yoon

Hematology/Oncology, Kyung Hee University Hospital, Seoul, Korea

H. Saito

Nagoya National Hospital, and

Aichi Blood Disease Research Foundation, Nagoya, Japan 


\section{Introduction}

The autosomal dominant macrothrombocytopenia with leukocyte inclusions, May-Hegglin anomaly (MHA) (May 1909; Hegglin 1945), Sebastian syndrome (SBS) (Greinacher et al. 1990), and Fechtner syndrome (FTNS) (Peterson et al. 1985), are rare human disorders characterized by a triad of giant platelets, thrombocytopenia, and characteristic Döhle body-like leukocyte inclusions. MHA and SBS can be differentiated by subtle ultrastructural leukocyte inclusion features (Greinacher et al. 1990), whereas patients with Fechtner syndrome suffer from a wide range of symptoms associated with Alport syndrome, including nephritis, deafness, and cataracts (Peterson et al. 1985). Biochemical studies have shown that platelet function and platelet membrane glycoproteins are normal in these disorders (Coller and Zarrabi 1981). Electromicroscopic analysis demonstrated that there is no significant morphological change in the platelets (Lusher et al. 1968; Jenis et al. 1971). A defect in the organization of the demarcation membranes of megakaryocytes during platelet formation has been suggested as a cause of giant platelets (Godwin and Ginsburg 1974). Döhle body-like leukocyte inclusions, another feature of the disorder, are suggested to be paracrystalline arrays of depolymerized ribosomes (Jenis et al. 1971). The underlying mechanisms for the pathogenesis of giant platelets as well as of leukocyte inclusions are unresolved.

By means of genome-wide linkage analysis, we first mapped a locus for the MHA gene on chromosome 22q12.3-q13.2 (Kunishima et al. 1999). Recently, we and others identified a gene mutated in the autosomal dominant macrothrombocytopenia with leukocyte inclusions by a positional candidate gene approach (The May-Hegglin/
Fechtner Syndrome Consortium 2000; Kelley et al. 2000; Kunishima et al. 2001). This gene, $M Y H 9$, encodes a large $(224 \mathrm{kDa})$ cytoplasmic protein, nonmuscle myosin heavy chain-A (NMMHC-A) (Toothaker et al. 1991; Saez et al. 1990). Structurally, NMMHC-A consists of an N-terminal motor/head domain, which contains ATP-binding and actin-binding domains, and a C-terminal tail domain, which undergoes dimerization to form a coiled-coil rod structure. Myosin is involved in the motor function for diverse movements such as cytokinesis, cell shape, and specialized functions such as secretion and capping (Sellers 2000). Although NMMHC-A has similar cellular functions in neutrophils and platelets (Maupin et al. 1994), its mechanism of action and/or the influence by gene defects in $M Y H 9$ on platelets and leukocytes are poorly understood.

To determine the incidence and pattern of $M Y H 9$ mutations and to correlate the location of mutations with clinical phenotypes, we studied 11 unrelated families and 3 sporadic cases with MHA, SBS, or FTNS. We detected the molecular defects in all 14 patients and found six novel mutations that include three missense and three deletion mutations.

\section{Materials and methods}

\section{Subjects}

We studied 11 families and three sporadic patients with the autosomal dominant macrothrombocytopenia with leukocyte inclusions, i.e., MHA, SBS, and FTNS. The patients were from Japan, Korea, and China. The diagnosis and hematological and clinical characteristics of the affected individuals are summarized in Table 1 . The mutations for 6

Table 1. Hematological and clinical characteristics of 20 patients with autosomal dominant macrothrombocytopenia with leukocyte inclusions

\begin{tabular}{|c|c|c|c|c|c|c|c|c|c|}
\hline $\begin{array}{l}\text { Family/ } \\
\text { case }^{\mathrm{a}}\end{array}$ & Diagnosis $^{\mathrm{b}}$ & Inheritance & $\begin{array}{l}\text { Ethnic } \\
\text { origin }\end{array}$ & $\begin{array}{l}\text { Giant } \\
\text { platelets }\end{array}$ & Thrombocytopenia & $\begin{array}{l}\text { Leukocyte } \\
\text { inclusions }\end{array}$ & Deafness & Nephritis & Cataracts \\
\hline 1 & MHA & Familial & Korea & + & + & + & - & - & - \\
\hline 2 & FTNS & Familial & Japan & + & + & + & + & + & + \\
\hline 3 & SBS & Familial & Japan & + & + & + & - & - & - \\
\hline 4 & SBS & Familial & Japan & + & + & + & - & - & - \\
\hline 5 & MHA/SBS & Sporadic & Japan & + & + & + & - & - & - \\
\hline 6 & SBS & Familial & Japan & + & + & + & - & - & - \\
\hline 7 & MHA & Familial & Japan & + & + & + & - & - & - \\
\hline 8 & MHA & Familial & Japan & + & + & + & - & - & - \\
\hline 9 & MHA & Familial & Japan & + & + & + & - & - & - \\
\hline 10 & MHA & Familial & Japan & + & + & + & - & - & - \\
\hline 11 & MHA/SBS & Sporadic & Japan & + & + & + & - & - & - \\
\hline 12 & MHA & Familial & China & + & + & + & - & - & - \\
\hline 13 & FTNS & Familial & Japan & + & + & + & + & + & - \\
\hline 14 & MHA & Familial & Korea & + & + & + & - & - & - \\
\hline 15 & MHA & Familial & Japan & + & + & + & - & - & - \\
\hline 16 & SBS & Familial & Japan & + & + & + & - & - & - \\
\hline 17 & MHA & Familial & Japan & + & + & + & - & - & - \\
\hline 18 & MHA & Familial & Korea & + & + & + & - & - & - \\
\hline 19 & SBS & Sporadic & Japan & + & + & + & - & - & - \\
\hline 20 & MHA & Familial & Japan & + & + & + & - & - & - \\
\hline
\end{tabular}

MHA, May-Hegglin anomaly; FTNS, Fechtner syndrome; SBS, Sebastian syndrome

${ }^{a}$ Families 7, 8, 10, 15, 16, and 17 have been previously reported by Kunishima et al. (2001)

${ }^{\mathrm{b}} \mathrm{MHA} / \mathrm{SBS}$ indicates that the diagnosis was made without electromicroscopic examinations 
Japanese families $(7,8,10,15,16$, and 17) have been reported previously (Kunishima et al. 2001). The diagnosis was initially made by hematologists from each country based on hemato-morphological examinations that detected a triad of giant platelets, thrombocytopenia, and leukocyte inclusions. All the blood smears were reexamined and confirmed by another hematologist. In addition, family members of each patient were examined for the presence or absence of abnormal hemostasis, renal diseases, or hearing disability. All patients had thrombocytopenia, giant platelets, and leukocyte inclusions (Table 1). For each mutation in the MYH9 gene, genomic DNA from 100 healthy Japanese individuals was screened and served as a control for gene polymorphism. Each individual gave informed consent for the study and the study was approved by the institutional ethical committee.

\section{Amplification of MYH9}

Genomic DNA was extracted from peripheral blood leukocytes or from peripheral blood spots made on FTA Gene Cards (GibcoBRL, Life Technologies, Rockville, MD, USA) according to a standard phenol/chloroform extraction. The entire sequence of 40 coding exons and exonintron boundaries of $M Y H 9$ was amplified by polymerase chain reaction (PCR) (Dunham et al. 1999). The primers used for PCR amplification and DNA sequence analysis are listed in Table 2. PCR amplification proceeded in a thermal cycler (GeneAmp PCR System 9600, Perkin-Elmer Cetus, Norwalk, CT, USA) for 35 cycles of $30 \mathrm{sec}$ at $95^{\circ} \mathrm{C}, 30 \mathrm{sec}$ at $55^{\circ} \mathrm{C}-60^{\circ} \mathrm{C}$, and $45-60 \mathrm{sec}$ at $72^{\circ} \mathrm{C}$ with AmpliTaq Gold DNA polymerase (PE Applied Biosystems, Foster City, CA, USA). Amplified DNA fragments were purified and subjected to direct cycle sequence analysis using a Thermo Sequenase II dye terminator cycle sequencing kit (Amersham Pharmacia Biotech, Buckinghamshire, UK) on an ABI 373A DNA sequencer. The $M Y H 9$ cDNA sequence was numbered, as the first ATG is +1 and amino acid residues are numbered from the ATG initiation codon (residue 1) (Toothaker et al. 1991; Saez et al. 1990).

\section{Mutational analysis}

To screen for nine reported MYH9 mutations (The MayHegglin/Fechtner Syndrome Consortium 2000; Kelley et al. 2000; Kunishima et al. 2001), the eight primer pairs shown in Table 3 were used to amplify exons 1, 16, 25, 26, 30, 38, and 40 from genomic DNA from the patients. All amplified PCR fragments were subjected to restriction fragment length polymorphism (RFLP) analysis. To screen the muta-

Table 2. Primers used for PCR-DNA sequence analysis of $M Y H 9\left(5^{\prime}>3^{\prime}\right)$

\begin{tabular}{lllc}
\hline Exon & Sense & Antisense & Product (bp) \\
\hline 1 & GTGATCTTGTGTGGCTGACG & CTTCTCAACCAGAGAGCCAG & 527 \\
2 & CCTTCACATCAGGCTGACTC & CCATCACCAGCCACTAGATC & 390 \\
3 & CATCTGTGACACTGTGCTCC & TCAAGAATGAGAACAGACTG & 199 \\
4 & CAACAGAATGCGAGGCAGTG & GTGCTCTTCCTCCATCATCC & 259 \\
5 & ACGTTGGACATGCTGACACG & AGATAGCACCGAGTCTGAAC & 325 \\
6 & TCTCTAGCTCCCCATGTCAG & TGGAATCAGGAGGCAGCTTC & 207 \\
7 & AAAGTGGAGGGCTTGCCTTC & GATGTCTACGGTCCAATTCT & 230 \\
8 & CCAACCTTTGAGGTCACAGC & CCATACACTGAAGGCCTTCC & 361 \\
9 & GCTTGTCTGGCTTGAGGATC & CTCCTGAGCAAATCCATGGC & 240 \\
10 & TGCTCCCAAGTCAAGCAGAG & TGAAAGTGCCTGACACGGAC & 343 \\
11 & CTTAGGGCTGTTCCTCTAGG & CAGATCGATGCAGGACCATG & 370 \\
12 & TCTTTAGTGCAGGGTTGGCG & CAACACAGAGCTGAGGTGAG & 309 \\
13 & CTGTGGGATTCAGGGGATTC & ACTCCACCTCTCCTGTGAAG & 331 \\
14 & AGACCCTGTGAGCACACATG & TGTGGAGGTGGGAAGATGAC & 244 \\
15 & TCCTGTTGTTTCATTCTGTCTC & GACTGAAGGCTCTGTGCATG & 438 \\
16 & TTGCCCTGTCAGGTTCATAG & CCTCTGGGACTCACTGCAC & 276 \\
17 & TATTCCAGGCATGTGGAATCG & ACCACTGATATAGCAAGGTGG & 227 \\
18 & TTCTGCTCTCCAGATGCTCG & GCTAAGTGCCCTTTGGCAAC & 367 \\
19 & AGACCAGGACTGTTAGGTCG & TTAGCCAGGTATGTATGGTGG & 244 \\
20 & ACTTTCATGTTCCAGAGGTG & TGCAGAGAGACTGGTCACTG & 389 \\
21 & CTTGAGGATGGAGTGGCTTG & GACCCTAATTCCATGTTCTCC & 367 \\
22 & CTCCTTGGCGTTTGGATCTG & GCAGAAGAGACAGGAAGCAG & 347 \\
23 & GGCTAGCAAGTGTTCTGTGC & AGTGCTGTAGTGTGACCCAG & 317 \\
24 & ATGGCACTGAGGGCTATGTG & TGCTCACAGCTCACTAGTGC & 308 \\
25 & TGTCCTGCAAACTCTGCTCC & GTCCATGTCTCCAAGCCAAG & 351 \\
26 & CTCTTTGGCAGGGAAGAGC & AGAACCCTGCAACTGCTCTG & 323 \\
27,28 & GTGATGATAGACCAGCCAGC & GAAGGAGAGGATGGGCAATC & 712 \\
29 & TTGGACACCACCCTCCTAAG & CCTTGAGAGCACTGATGTGG & 361 \\
30 & ATAACTGGGCAGATCCCTGG & TTGCTTTGACTCAGTGCTTG & 438 \\
31,32 & TCCCATCATGAGGATGCACG & AGAGAACAGAAGCCTGCGTG & 827 \\
33 & TGAGTTCAGAGCTAGGGCAG & GCACCTTCATATGTAGTTGGC & 299 \\
34 & AGCATTGAGTGGAGCACCAG & TATGTAACCTGAGAGCCATCC & 375 \\
$35-37$ & GAGCTAGAGGGTTTCTGGAG & AGACAGAGAGCTGGTTGTGG & 1073 \\
38,39 & TCCTGGTTAGGGCTTGTTGG & TGGTGACATTCGTGCCTTGC & 591 \\
40 & TTGAGATGTGTGGGCTGTGC & GAGGCATGTTCACAGCAGTC & 310 \\
\hline & & & \\
& & & \\
& & &
\end{tabular}




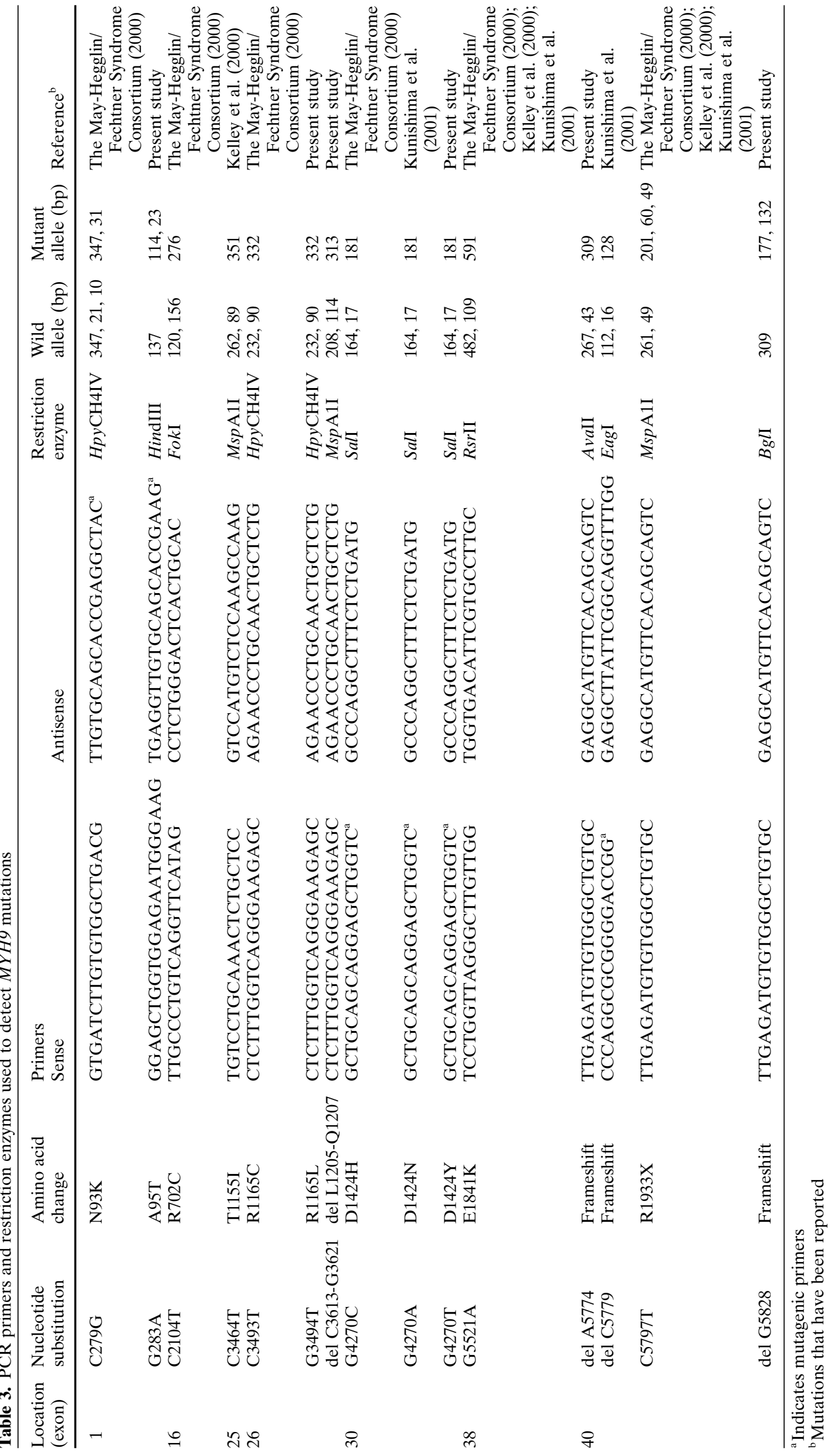


tions located in a neighboring position, the same primer pairs were used and different restriction enzymes were selected for analysis (Table 3). For detecting D1424H and D1424N, the same primer pairs were used. Based on the results obtained in this study, two additional primer pairs were designed for A95T and delA5774. If none of these mutations were detected, sequencing of the entire coding exons was performed by using the primer pairs listed in Table 2. All mutations were verified by re-amplifying from genomic DNA and then repeating RFLP analysis or sequencing.

\section{Reverse transcriptase (RT) - PCR}

Total platelet RNA was extracted using the Catrimox reagent (Takara Shuzo, Otsu, Japan) and first-strand cDNA was synthesized using ThermoScript RT and oligo-dT primer (GibcoBRL) (Kunishima et al. 1998). PCR amplification was performed on the cDNA with appropriate primers for the $M Y H 9$ cDNA sequence followed by sequencing and RFLP analysis.

\section{Immunofluorescence analysis}

For immunofluorescence staining, peripheral blood samples were smeared on glass slides and air dried. After being permeabilized with acetone, the cells were hydrated and incubated with anti-NMMHC-A polyclonal antibody (Biomedical Technologies Inc., Stoughton, MA, USA). Slides were then incubated with rhodamine-labeled swine antirabbit IgG (DAKO, Glostrup, Denmark). The stained cells were analyzed by confocal laser scanning microscopy (MRC-1024, Bio Rad, Richmond, CA, USA) (Kunishima et al. 2001).

\section{Results}

Detection of MYH9 mutations in patients with the autosomal dominant macrothrombocytopenia with leukocyte inclusions

The results obtained from our previous study (Kunishima et al. 2001) and the current study are summarized in Table 4. At first, we looked for the nine previously reported $M Y H 9$ mutations by PCR-RFLP analysis in 14 patients. We identified three families with R1165C, two with E1841K, and one with R1933X. In sporadic cases, we found one patient with E1841K and one with R1933X. Each mutation was confirmed by sequencing. In the remaining five families and one sporadic patient, none of the nine reported mutations was detected and these patients were investigated for new mutations. In each patient, all 40 coding exons and their flanking intronic sequences were amplified and directly sequenced. As a result, heterozygous $M Y H 9$ mutations were identified in all five families and one sporadic patient. Three novel missense mutations, A95T, R1165L, and D1424Y, were found in family 1 , sporadic case 5 and family 9 , respectively. In families 14 and 20, one-base deletions, del A5774 and del G5828, were detected, respectively. Both deletion mutations result in a frameshift and premature termination. In each family, the corresponding mutation cosegregated with the disease phenotype as macrothrombocytopenia with

Table 4. Identified $M Y H 9$ mutations in 20 patients with autosomal dominant macrothrombocytopenia with leukocyte inclusions

\begin{tabular}{|c|c|c|c|c|c|c|}
\hline $\begin{array}{l}\text { Family/ } \\
\text { case }^{a}\end{array}$ & Diagnosis $^{\mathrm{b}}$ & Exon & substitution & $\begin{array}{l}\text { Nucleotide } \\
\text { change }\end{array}$ & $\begin{array}{l}\text { Amino acid } \\
\text { Transcription }^{c}\end{array}$ & Immunofluorescence ${ }^{\mathrm{d}}$ \\
\hline 1 & MHA & 1 & G283A & A95T & ND & ND \\
\hline 2 & FTNS & 26 & $\mathrm{C} 3493 \mathrm{~T}$ & $\mathrm{R} 1165 \mathrm{C}$ & + & + \\
\hline 3 & SBS & 26 & C3493T & $\mathrm{R} 1165 \mathrm{C}$ & + & + \\
\hline 4 & SBS & 26 & $\mathrm{C} 3493 \mathrm{~T}$ & $\mathrm{R} 1165 \mathrm{C}$ & + & + \\
\hline 5 & MHA/SBS & 26 & G3494T & $\mathrm{R} 1165 \mathrm{~L}$ & + & + \\
\hline 6 & SBS & 26 & del C3613-G3621 & del L1205-Q1207 & + & + \\
\hline 7 & MHA & 30 & G4270C & D1424H & + & + \\
\hline 8 & MHA & 30 & G4270A & D1424N & + & + \\
\hline 9 & MHA & 30 & G4270T & D1424Y & ND & ND \\
\hline 10 & MHA & 38 & G5521A & $\mathrm{E} 1841 \mathrm{~K}$ & + & + \\
\hline 11 & MHA/SBS & 38 & G5521A & $\mathrm{E} 1841 \mathrm{~K}$ & + & + \\
\hline 12 & MHA & 38 & G5521A & E1841K & ND & ND \\
\hline 13 & FTNS & 38 & G5521A & E1841K & + & + \\
\hline 14 & MHA & 40 & del A5774 & Frameshift & ND & ND \\
\hline 15 & MHA & 40 & del C5779 & Frameshift & + & + \\
\hline 16 & SBS & 40 & C5797T & R1933X & + & + \\
\hline 17 & MHA & 40 & C5797T & R1933X & + & + \\
\hline 18 & MHA & 40 & C5797T & R1933X & ND & ND \\
\hline 19 & SBS & 40 & C5797T & R1933X & + & + \\
\hline 20 & MHA & 40 & del G5828 & Frameshift & + & + \\
\hline
\end{tabular}

${ }^{a}$ Families 7, 8, 10, 15, 16, and 17 have been previously reported by Kunishima et al. (2001)

${ }^{\mathrm{b}} \mathrm{MHA} / \mathrm{SBS}$ indicates that the diagnosis was made without electromicroscopic examinations

${ }^{c}+$, presence of transcription of the mutant MYH9 allele. ND, not determined

${ }^{\mathrm{d}}+$, abnormal localization of NMMHC-A in leukocytes. ND, not determined 
leukocyte inclusion bodies. In sporadic patients, only the affected individual had a mutation and none of their parents had mutations. Nonpaternity in each of the sporadic cases was excluded by genotyping. All mutations were screened in 100 normal individuals and none was found.

Overall, MYH9 mutations were identified in all 14 families/sporadic patients. All patients had a heterozygous MYH9 mutation. R1165C, E1841K, and R1933X were the most common mutations and they covered $55 \%$ (11 of 20 families/sporadic patients) of genetic defects in patients examined (Table 4).

To determine the effects of the mutations on $M Y H 9$ expression, we investigated the presence of an mRNA transcript in platelets from the available patients using RT-PCR analysis. The results of this analysis confirmed our previous data that each mutant allele was expressed at the mRNA level in the patients' platelets (Table 4) (Kunishima et al. 2001).

\section{Polymorphisms}

During the course of the DNA sequence analysis of the complete $M Y H 9$ of the patients and control subjects, a number of novel base changes, as compared to the published sequence, were identified. The intronic base changes were excluded from being the genetic defect underlying the disease when they were not located in or near a consensus sequence motif considered critical for RNA processing. They were therefore considered to be polymorphisms. Among the polymorphic substitutions, there was only one substitution that results in an amino acid change, $11625 \mathrm{~V}$ (A4874G). This substitution is not associated with, nor does it cause, the autosomal dominant macrothrombocytopenia with leukocyte inclusions because it was found in homozygous form in normal individuals. The allele frequency in 100 normal controls is 0.36 .

\section{Genotype-phenotype correlation}

We investigated the phenotypes of patients whose mutations had been identified after classifying the clinical phenotypes into the following two groups: (1) a typical phenotype as classical MHA and/or SBS, in which only platelets and leukocytes were affected; and (2) patients with Alport manifestations. All patients had thrombocytopenia, giant platelets, and leukocyte inclusions. R1165C mutation was found in SBS and FTNS. E1841K was detected in MHA and FTNS.

\section{Discussion}

This study presents the first systemic analysis of the $M Y H 9$ gene and clinical phenotypes in a cohort of patients with the autosomal dominant macrothrombocytopenia with leukocyte inclusions from Asian ethnic groups. All the patients were either from Japan, Korea, or China. To reduce the amount of sequencing necessary to identify individual mu- tations, we first used PCR-RFLP and nine known mutations were screened by using the specific primer pairs (Table 2). Mutations in 8 families/sporadic cases were identified by this method and direct sequencing of amplified PCR fragments led to characterization of the molecular defects in the remaining 6 families/sporadic cases. The combination of PCR-RFLP and PCR-direct sequencing analysis identified MYH9 mutations in all 11 families and 3 sporadic cases who were diagnosed with MHA, SBS, or FTNS (Table 4). For each family, all available family members were tested for the identified mutation.

MYH9 mutations that are pathognomonic or may be pathognomonic were distinguished from benign polymorphisms by the following criteria: (1) the mutations were not present in at least 100 normal subjects; (2) nonsense and frameshift mutations are considered pathognomonic; and (3) the molecular defects matched the phenotype in the family involved.

Six point mutations and three nucleotide deletions in the MYH9 gene were identified from all of the analyzed cases. Six mutations, A95T, R1165L, del L1205-Q1207, D1424Y, del A5774, and del G5828, were novel, whereas other mutations, R1165C, E1841K, and R1933X, have been shown to be identical by us (Kunishima et al. 2001) and others (The May-Hegglin/Fechtner Syndrome Consortium 2000; Kelley et al. 2000). The E1841K mutation was found in two Japanese families, in which unrelatedness was confirmed by haplotype analysis (data not shown). One sporadic case from Japan and one family from China also had the E1841K mutation. Two families and one sporadic case from Japan and one family from Korea shared the same nucleotide change at nucleotide C5797, resulting in the nonsense mutation at Arg1933. These mutations have also been found in Europe and the United States (The MayHegglin/Fechtner Syndrome Consortium 2000; Kelley et al. 2000). These observations suggest that there is no marked difference in the locations of mutations between different ethnic groups.

When the current results are combined with our previous report (Kunishima et al. 2001), the mutation detection rate was $100 \%$ for MHA, SBS, and FTNS (20/20). In the previous report, we detected mutations in six of seven families and failed to detect in only one family. The high detection rate for $M Y H 9$ mutations can be achieved by taking several analytical steps. First, we performed systematic screening for mutations with PCR-RFLP to detect known mutations followed by direct sequencing of the entire coding sequence, including exon-intron boundaries of the gene. Although convenient mutation screening methods such as single-strand conformation polymorphism can be applied to investigate the large $M Y H 9$ gene, the detection rate for base substitutions may not reach $100 \%$. Second, the precise diagnosis of each patient was important. The diagnosis for each patient was performed by careful hematological examinations, including the platelet/leukocyte morphology of May-Grünwald-Giemsa-stained, freshly prepared peripheral blood smears. Furthermore, we performed immunofluorescence analysis with anti-NMMHC-A antibody to confirm abnormal localization of NMMHC-A 
protein in granulocytes from all of the Japanese patients (Table 4).

There is evidence in mammalian cells that transcripts containing nonsense mutations undergo abnormal processing that may result in instability of mRNA (Frischmeyer and Dietz 1999). However, RT-PCR experiments confirmed our previous data (Kunishima et al. 2001), demonstrating that most mutant alleles harboring the nonsense mutation or frameshift mutations were expressed in the patients' platelets. Immunofluorescence studies revealed abnormal localization of the NMMHC-A protein in the leukocytes (Table 4).

It is also interesting to note that nonsense and frameshift mutations resulting in premature termination were found only in the last exon (exon 40). Because these nonsense and frameshift mutations reside in close proximity to the natural stop codon, they may not cause defects in transporting or processing, and the translated truncated proteins would retain contractile function without completely destroying the functionally important myosin heads. By self-association in its carboxyl-terminal domain, a myosin heavy chain forms the backbone of the thick myosin filament. Thus, the random association of wild-type and mutant polypeptides would suggest that the mutations in the rod domain have dominant negative effects. Indeed, all but one of the mutations was located in the rod domain of the $\mathrm{MYH}$ gene (Fig. 1). These findings suggest that mutations have an impact on the coiled-coiled rod domain in the pathogenesis of autosomal dominant macrothrombocytopenia with leukocyte inclusions.

Figure 2 shows that Ala95, Arg1165, Asp1424, and Glu1841 are highly conserved amino acid residues among the nonmuscle or smooth muscle myosin heavy chain superfamily, indicating the functional importance of the four residues in determining the structure and function of the protein. Asp1424 and Glu1841 appear to be frequently mutated. Three groups, including ours, have reported substitutions at Asp1424 to His or Asn and substitutions at Glu1841 to Lys (The May-Hegglin/Fechtner Syndrome Consortium 2000; Kelley et al. 2000; Kunishima et al. 2001). In this study, we found a novel Tyr mutation at Asp1424.

Although there is only one SBS case that harbors the R1165C mutation (The May-Hegglin/Fechtner Syndrome Consortium 2000), in the current study we found four cases with substitutions at Arg1165 (three Cys mutations and one Leu mutation, Fig. 1). A propositus of family 2 had an FTNS phenotype and others had an MHA or SBS phenotype. These observations may suggest that R1165C or R1165L are new common mutations, particularly found in Japanese patients with MHA, SBS, and FTNS.

Overall, these four positions, Arg1165, Asp1424, Glu1841, and R1933, are hotspots for mutation within the MYH9 coding region and represent $75 \%$ (15/20) of identified mutations from our two studies. Moreover, the muta-

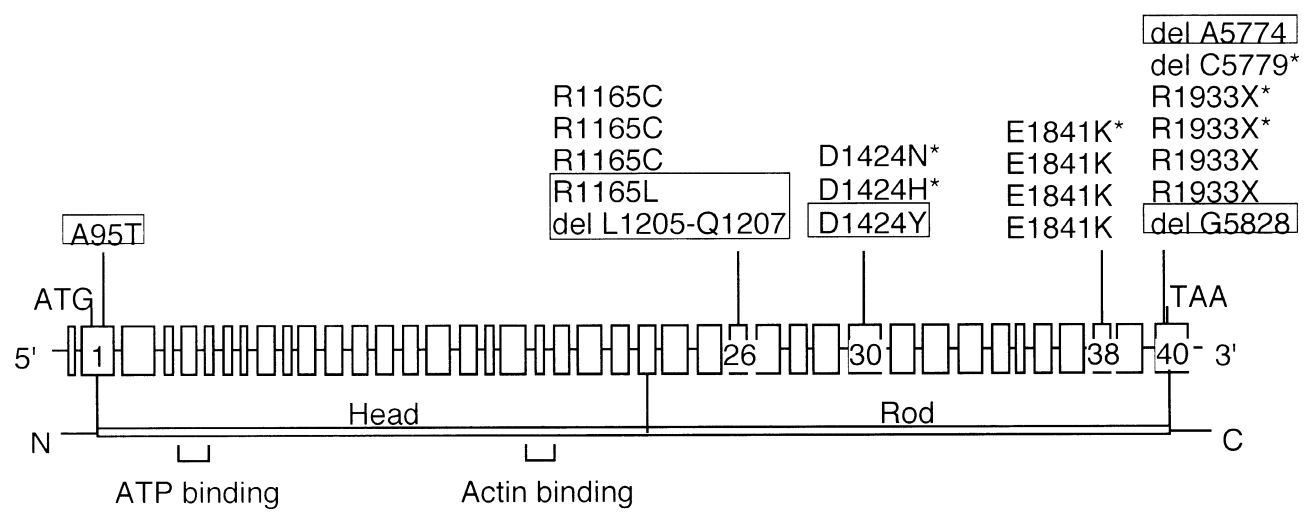

Fig. 1. The spectrum of $M Y H 9$ mutations in patients with the autosomal dominant macrothrombocytopenia with leukocyte inclusions. Schematic representations of one noncoding and 40 coding exons of MYH9 are shown at the top, and the predicted NMMHC-A protein at the bottom. The amino-terminal globular head domain and the carboxy-terminal rod domain are shown. The transcription initiation codon $(A T G)$, natural stop codon (TAA), ATP-binding domain, and actin-binding domain are indicated. Novel mutations identified in this study are boxed. The previously reported mutations in six Japanese families (Kunishima et al. 2001) are indicated by an asterisk
Fig. 2. NMMHC-A sequence alignment. Amino acid sequence alignment is shown from the known NMMHC-A from the other species and human NMMHC-B isoform. The identified missense mutations are indicated in bold

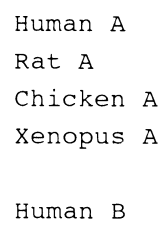

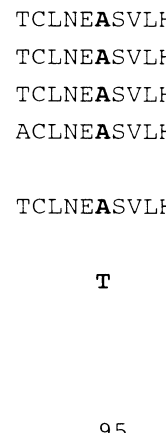

95

ELRSKREQEVN
ELRSKREQEVS
ELRSKREQEVT
ELRTKREQEVT
ELRTKREQEVA
C
L
1165

OOELDDLLVDL QQELDDLTVDL QOELDDIAVDL QQELDDISVDL

QQELDDLTVDL
QVRRTEKKLKD QVRRAEKKLKD QVRRAEKKLKD QVRRTEKKLKD

LVRRTEKKLKE
$\mathrm{H}$

$\mathrm{Y}$ 
tions are found in several western and Asian ethnic groups, indicating a universal origin of this complex disease.

From the combined observations of our two studies and other studies (The May-Hegglin/Fechtner Syndrome Consortium 2000; Kelley et al. 2000), we have determined that the clinical phenotype of individuals sharing the same mutation can be variable in terms of the development of Alport syndrome (Table 4). The phenotype of patients with mutations at Arg1165 is variable, because a propositus of family 2 had an FTNS phenotype but three other cases had an MHA or SBS phenotype. A patient reported by the MayHegglin/Fechtner Syndrome Consortium showed an SBS phenotype (The May-Hegglin/Fechtner Syndrome Consortium 2000). The phenotype of patients bearing mutations at Asp1424 also appears to be variable. One case reported by the May-Hegglin/Fechtner Syndrome Consortium has FTNS (The May-Hegglin/Fechtner Syndrome Consortium 2000), but our three cases have MHA (Table 4). The phenotype of patients with mutations at Glu1841 has been reported to be all MHA or MHA/SBS (The May-Hegglin/ Fechtner Syndrome Consortium 2000; Kelley et al. 2000; Kunishima et al. 2001), but our family 13 had a member with an E1841K mutation who had developed Alport manifestations. In contrast, a total of eight R1933X mutation cases have been reported, and none showed an additional extrahematological syndrome like deafness or nephritis (The May-Hegglin/Fechtner Syndrome Consortium 2000; Kelley et al. 2000; Kunishima et al. 2001). These facts may indicate that these four frequent mutations are not necessarily responsible for the development of Alport syndrome. Further genetic or epigenetic unknown factors may underlie the distinct expression of clinical symptoms. In the future, the identification of new mutations may reveal their relative involvement in the expression of Alport Syndrome.

Acknowledgments We thank Drs Masaki Yamaguchi, Masahito Tsurusawa, and Hiroyuki Sakatoku for patients' samples and Drs C. Inoue and K. Ozawa for support. This study was supported by Grantsin Aid for Scientific Research (10557090, 11470209, and 12670981) from the Ministry of Education, Science, Sports and Culture, and for Research on Specific Diseases from the Ministry of Health and Welfare.

\section{References}

Coller BS, Zarrabi MH (1981) Platelet membrane studies in the MayHegglin anomaly. Blood 58:279-284
Dunham I, Shimizu N, Roe BA, Chissoe S, Hunt AR, Collins JE, Bruskiewich R, Beare DM, Clamp M, Smink LJ, Ainscough R, Almeida JP, Babbage A, Bagguley C, Bailey J, Barlow K, Bates KN, Beasley O, Bird CP, Blakey S, Bridgeman AM, Buck D, Burgess J, Burrill WD, O'Brien KP (1999) The DNA sequence of human chromosome 22. Nature 402:489-495

Frischmeyer PA, Dietz HC (1999) Nonsense-mediated mRNA decay in health and disease. Hum Mol Genet 8:1893-1900

Godwin HA, Ginsburg AD (1974) May-Hegglin anomaly: a defect in megakaryocyte fragmentation? Br J Haematol 26:117-128

Greinacher A, Nieuwenhuis HK, White JG (1990) Sebastian platelet syndrome: a new variant of hereditary macrothrombocytopenia with leukocyte inclusions. Blut 61:282-288

Hegglin R (1945) Gleichzeitige konstitutionelle Veranderungen an Neutrophilen und Thrombocyten. Helv Med Acta 12:439-440

Jenis EH, Takeuchi A, Dillon DE, Ruymann FB, Rivkin S (1971) The May-Hegglin anomaly: ultrastructure of the granulocytic inclusion. Am J Clin Pathol 55:187-196

Kelley MJ, Jawien W, Ortel TL, Korczak JF (2000) Mutation of MYH9, encoding non-muscle myosin heavy chain A, in MayHegglin anomaly. Nat Genet 26:106-108

Kunishima S, Mizuno S, Naoe T, Saito H, Kamiya T (1998) Genes for thrombopoietin and $\mathrm{c}-\mathrm{mpl}$ are not responsible for familial thrombocythaemia: a case study. Br J Haematol 100:383-386

Kunishima S, Kojima T, Tanaka T, Kamiya T, Ozawa K, Nakamura Y, Saito H (1999) Mapping of a gene for May-Hegglin anomaly to chromosome 22q. Hum Genet 105:379-383

Kunishima S, Kojima T, Matsushita T, Tanaka T, Tsurusawa M, Furukawa Y, Nakamura Y, Okamura T, Amemiya N, Nakayama T, Kamiya T, Saito H (2001) Mutations in the NMMHC-A gene cause autosomal dominant macrothrombocytopenia with leukocyte inclusions (May-Hegglin anomaly/Sebastian syndrome). Blood 97:11471149

Lusher JM, Schneider J, Mizukami I, Evans RK (1968) The MayHegglin anomaly: platelet function, ultrastructure and chromosome studies. Blood 32:950-961

Maupin P, Phillips CL, Adelstein RS, Pollard TD (1994) Differential localization of myosin-II isozymes in human cultured cells and blood cells. J Cell Sci 107:3077-3090

May R (1909) Leukozyteneinschlusse. Dtsch Arch Klin Med 96:1-6

The May-Hegglin/Fechtner Syndrome Consortium (2000) Mutations in MYH9 result in the May-Hegglin anomaly, and Fechtner and Sebastian syndromes. Nat Genet 26:103-105

Peterson LC, Rao KV, Crosson JT, White JG (1985) Fechtner syndrome - a variant of Alport's syndrome with leukocyte inclusions and macrothrombocytopenia. Blood 65:397-406

Saez CG, Myers JC, Shows TB, Leinwand LA (1990) Human nonmuscle myosin heavy chain mRNA: generation of diversity through alternative polyadenylylation. Proc Natl Acad Sci USA 87:1164-1168

Sellers JR (2000) Myosins: a diverse superfamily. Biochim Biophys Acta 1496:3-22

Toothaker LE, Gonzalez DA, Tung N, Lemons RS, Le Beau MM, Arnaout MA, Clayton LK, Tenen DG (1991) Cellular myosin heavy chain in human leukocytes: isolation of $5^{\prime}$ cDNA clones, characterization of the protein, chromosomal localization, and upregulation during myeloid differentiation. Blood 78:1826-1833 